\title{
Towards a Strategic Approaches in Alternative Tests for Pesticide Safety
}

\author{
Yoonjeong Jang ${ }^{1}$, Ji-Eun Kim ${ }^{1}$, Sang-Hee Jeong ${ }^{2}$ and Myung-Haing Cho ${ }^{1,3,4,5}$ \\ ${ }^{1}$ Laboratory of Toxicology, BK21 PLUS Program for Creative Veterinary Science Research, \\ Research Institute for Veterinary Science and College of Veterinary Medicine, Seoul National University, Seoul, Korea \\ ${ }^{2}$ Department of Bio Applied Toxicology, Hoseo Toxicology Research Center, Hoseo University, Asan, Korea \\ ${ }^{3}$ Graduate School of Convergence Science and Technology, Seoul National University, Suwon, Korea \\ ${ }^{4}$ Graduate Group of Tumor Biology, Seoul National University, Seoul, Korea \\ ${ }^{5}$ Advanced Institute of Convergence Technology, Seoul National University, Suwon, Korea
}

(Received June 24, 2014; Revised September 19, 2014; Accepted September 20, 2014)

\begin{abstract}
Pesticides have provided significant benefits including plant disease control and increased crop yields since people developed and utilized them. However, pesticide use is associated with many adverse effects, which necessitate precise toxicological tests and risk assessment. Most of these methods are based on animal studies, but considerations of animal welfare and ethics require the development of alternative methods for the evaluation of pesticide toxicity. Although the usage of laboratory animals is inevitable in scientific evaluation and alternative approaches have limitations in the whole coverage, continuous effort is necessary to minimize animal use and to develop reliable alternative tests for pesticide evaluation. This review discusses alternative approaches for pesticide toxicity tests and hazard evaluation that have been used in peer-reviewed reports and could be applied in future studies based on the critical animal research principles of reduction, replacement, and refinement.
\end{abstract}

Key words: Pesticides, Alternative approach, Toxicological methods, Pesticides evaluation

\section{INTRODUCTION}

According to Korean agricultural chemicals regulation law, pesticides include germicides, insecticides, and herbicides used to prevent disease and agricultural pests such as germs, insects, nematodes, mites, viruses and weeds that can damage to agricultural produce(1). Pesticides are essential to control disease and pests to increase food production and improve plant breeds on limited farmland. However, there are sensitive harmful effects from using pesticides. A notable example is pesticide residue. Concern for public health has been raised because of remaining pesticides in foods and environmental contamination is caused by the spraying of agricultural chemicals. Therefore, it is important to test the toxicity of pesticides before and after their

Correspondence to: Myung-Haing Cho, Laboratory of Toxicology, College of Veterinary Medicine, Seoul National University, Seoul 151-742, Korea

E-mail: mchotox@snu.ac.kr

This is an Open-Access article distributed under the terms of the Creative Commons Attribution Non-Commercial License (http:// creativecommons.org/licenses/by-nc/3.0) which permits unrestricted non-commercial use, distribution, and reproduction in any medium, provided the original work is properly cited. release into the environment.

To assess the impact of pesticides on human health, toxicological classification must to be performed. Most toxicity tests for pesticides determine via oral exposure, application to the skin and the respiratory system. Among these, based on the result of acute oral toxicity test, the potency of toxicity is classified. Acute oral toxicity test is performed with two species of animals and they are administered orally. After $48 \mathrm{hrs}$, in accordance with median lethal dose $\left(\mathrm{LD}_{50}\right)$, which causes death in half of exposed test animals, pesticides are categorized as extremely hazardous, highly hazardous, moderately hazardous, or slightly hazardous (2).

In order to calculate $\mathrm{LD}_{50}$ values and test skin irritation, a majority of laboratory animals are employed. At present, people show ethical concerns and severe opposition to traditional toxicity tests. Since the $3 \mathrm{R}$ principle - reduction, replacement, and refinement - was proposed by B. Russell and R. Burch in 1959 (3), alternative approaches have been developed based on ethical considerations of animal welfare. Internationally, technical development of in vitro tests using cultured cells and in silico computer analysis has progressed satisfactorily to allow these tests to be considered as alternatives to in vivo tests using laboratory animals.

For instance, since 2009, the European Union has prohib- 
ited the production and sale of cosmetics tested on animals. And the European Center for Validation of Alternative Methods (ECVAM) has been established to develop nonanimal tests and to assess the reliability and relevance of tests for European regulatory mandates (4). Additional organizations created for developing and validating alternative methods include the Interagency Coordinating Committee for Validation of Alternative Methods (ICCVAM, USA), the Zentralstelle zur Erfassung und Bewertung von Ersatzund Ergänzungsmethoden zum Tierversuch (ZEBET, Germany), and Korean Center for the Validation of Alternative Methods (KoCVAM, Korea).

In Korea, animal protection law was amended completely in 2007 and the Ministry of Agriculture, Food and Rural Affairs prepared a legal basis for the principles of animal tests and Institutional Animal Care and Use Committee (IACUC). Clause 13 of the animal protection law covers the principles of animal testing based on replacement, reduction, and refinement. Under clause 25 , which delineates the constitution of the IACUC, the chief of an institution conducting animal experiments must constitute and operate an IACUC to protect animals and ensure that they are handled ethically. To experiment with animals, study plans must be considered by the IACUC, which can require actions to be taken for the protection of the animals used in the experiments (5). The Rural Development Administration (RDA) of Korea has also amended the 'Standard for Registration of Pesticides', which introduces methods to reduce experimental animal use and shift experiments to artificial cell lines, in accordance with the goal of maximizing animal welfare (6).

In 2010, Korea was the fifth in the world to join the 'International Cooperation on Alternative Test Methods' initiative, which focuses on the validation of alternative testing methods. The alternative tests introduced include acute oral toxicity and skin sensitization tests that use fewer animals than the traditional tests. For example, the traditional acute oral toxicity test uses approximately 50 rats whereas the alternative test can evaluate toxicity using 6-12 rats. The existing skin sensitization test uses a minimum of 30 guinea pigs and takes over a period of 20 days, whereas the alternative test replaces guinea pigs with mice, reduces the number of animals to fewer than 20, and shortens the tests duration to less than 8 days. The RDA concluded that there is little difference in safety between existing test methods and animal alternative tests (7).

Many tests for evaluating the toxicity of pesticides have been performed since agricultural chemicals were developed. This review will discuss materials related to toxicological tests of agricultural chemicals and published studies using alternative methods categorized as in vitro, in vivo and in silico. The endless endeavor of scientists developing alternative toxicity tests suggests a positive outlook for pesticide usage and protection of animals and ecosystems.

\section{CURRENT ISSUES IN TOXICOLOGICAL TESTS FOR PESTICIDES}

Residues from pesticides have had negative consequences for humans and the environment since their introduction. Pesticides can be exposed to the manufacturers and farm workers as well as the general population. Non-occupational exposure may occur through the use of improper hazardous pesticides in cities, public parks, golf club, and other environments such as swimming pools, and this exposure has a potential harmful influence on public health. When pesticides contaminate soil, rivers, and oceans, they may be able to produce adverse effects on non-target ecosystems. Therefore, it is urgent to measure pesticide exposure and to evaluate the toxicity level in humans, animals, and the environment.

Among the impacts on the ecosystem, the concern of harmful effect on honeybees has been evoked recently. Since 2007, researchers have increased their efforts to find a cause for the colony collapse disorder (CCD) phenomena which has decimated bee colonies. The issue is very complex, with many potential contributing factors, including parasites, pesticides, and stress. However, pesticides have consistently been implicated as a key issue in pollinator decline, through acute toxicity causing death and through sublethal exposure causing changes in bee reproduction, navigation, and foraging (8). Many scientific inferences have discussed the potential roles that pesticides applied to crops may play in honeybee health. Although no specific pesticide has been clearly associated with CCD, there are some evidences for the additive and synergistic effects of multiple pesticides on harming bees (9). Despite the importance of honeybees, the effects of pesticide exposure on colony health have not been systematically monitored, and the US Environmental Protection Agency (EPA) does not require data on sublethal effects for pesticide registration. The use of newer systemic pesticides, including neonicotinoids and phenylpyrazoles has become prevalent in the USA and these compounds are also present in plant tissues. Honeybees' exposure to these compounds is very different from that of traditional pesticides, where acute toxicity is a primary concern. Instead, honeybees at all stages of development may be chronically exposed to sublethal doses of these compounds. Restricting the use of new compounds may result in a reversion to older chemistries that are known to harm bees (9). Studies concerned with the potential impact of pesticides on beneficial organisms, including bees, are needed to influence the effective policymaking that ensures the long-term survival of the species and its economic impact.

Former pesticide toxicity testing methods required the use of large numbers of laboratory animals for $\mathrm{LD}_{50}$ calculation. Therefore, new methods have been developed that drastically reduce the number of animals compared to traditional methods. Two representative examples are the new 
acute oral toxicity test guidelines in the OECD Guidelines for the Testing of Chemicals, Test Guideline 420 and Test Guideline 423.

Test Guideline 420 is a refined approach to acute toxicity testing that was suggested by the British Toxicology Society in 1984 based on the administration of compounds at a series of fixed dose levels. The statistical properties of the Fixed Dose Procedure have been evaluated using mathematical models in a series of studies. In this procedure, groups of animals of a single sex are dosed in a stepwise procedure using the fixed doses of 5, 50, 300 and $2000 \mathrm{mg} /$ $\mathrm{kg}$. The initial dose level is selected as the dose expected to produce some signs of toxicity without causing severe toxic effects or mortality on the basis of a sighting study. Clinical signs and conditions associated with pain, suffering, and impending death, are described in detail in a separate OECD guidance document. This procedure continues until the dose causing evident toxicity or no more than one death is identified, when no effects are seen at the highest dose or when deaths occur at the lowest dose (10).

Test Guideline 423 is an acute stepwise toxicity testing procedure that uses 3 animals of a single sex per step. Depending on the mortality and/or the moribund status of the animals, 2 4 steps are usually necessary for evaluating the acute toxicity of the test substance. The method, as adopted in 1996, was extensively validated in vivo against $\mathrm{LD}_{50}$ data obtained from the literature, both nationally and internationally (11). These procedures are reproducible, use very few animals, and are able to rank.

New methods of testing the toxicity of agricultural chemicals are quicker and cost less than previous method. Experimental comparison between existing methods and new alternative methods will allow for toxicological classification of pesticides with respect to economic considerations and animal welfare concerns. Because pesticides are extensively utilized, policy decisions regarding, regulation and risk assessment are very important. Pesticide-related laws, management programs, and institutions have supported the development and experimenting of alternative testing methods around the world. Adherence to good laboratory practices (GLPs) provided in OECD guidelines minimizes overlapping experiments by facilitating the sharing of experimental results and laboratory techniques. Developed countries that are party to the OECD require experimental results obtained by GLP test agencies as toxicity test reports for all pesticides. In Korea, the RDA manages GLP system related to toxicity test of pesticides. Methods of pesticides testing are performed according to the standards and methods of registration of pesticides notified by the RDA, and foreign test standards such as those of the OECD and US EPA can also be accepted interchangeably (12).

Many nations have enacted and amended laws regulating agrochemicals. In Korea, agricultural chemicals regulation law was enacted and promulgated in 1957 and recently amended in 2014 to improve environmental conservation and public health by setting regulations regarding the manufacturing, import, safe usage, and handling of pesticides (1). In the European Union (EU), legislation on pesticides is found in regulation EU regulation 283 and 284, which sets safety data requirements for active substances and plant protection products (13). In the United States, registration, regulation, and risk assessment of pesticides follow guidelines in the Federal Insecticide, Fungicide, and Rodenticide Act (FIFRA) that are enforced by the U.S. EPA(14).

For the improved protection of human health and the environment, the U.S. EPA is developing and evaluating new molecular, cellular, and computational methods to supplement or replace more traditional methods of toxicity testing and risk assessment. The EPA's Pesticide Program prospect the results in computer-aided methods for better prediction of potential hazards and exposure amount, minimization of the number of animals, improved understanding of toxicity pathways, and search for diagnostic biomonitoring and surveillance methods to detect chemical exposures (15).

Furthermore, international harmonization of test guidelines by the OECD for chemicals/pesticides and the veterinary medicine, International Conference on Harmonization (ICH and VICH) have contributed in minimizing duplicate testing, probably save more animals than any single alternative method. International harmonization is as important as economic harmonization (16).

\section{IN VITRO ALTERNATIVE PESTICIDE TOXICITY TESTING METHODS}

In vitro (meaning "in glass" in Latin) study is experimentation with samples of tissue or cells under artificial conditions and outside of living organisms. In vitro testing procedures are important alternative agrochemical toxicity testing methods that meet the criteria of reduction, replacement, and refinement. Furthermore, in vitro tests offer several advantages over traditional in vivo methods, including controlled testing conditions, a high level of standardization, a reduction in variability between experiments, the absence of systemic effects, low cost, a small amount of required material, a limited amount of toxic waste, and reduced need for animals (17).

Each fields of toxicity studies have developed and used for investigation of agrochemicals. Several assays are used to predict pesticide-induced immunotoxic effects. The effects of persistent exposure to pesticide chemicals on the integrity of the immune system have recently drawn considerable interest as an additional indicator of potential problems. Pistl et al. (18) aimed to determine the action of several pesticides of different chemical structures with regard to biological effects on the function of phagocytes and lymphocytes isolated from the peripheral blood of 
sheep under in vitro conditions by using the iodo-nitro-tetrazolium reductase test and the leukocyte migration-inhibition assay. Assays for immunotoxicity must consider studies on humoral, cell-mediated immunity, leukocytes and nonspecific immunity.

Some widely used agrochemicals are suspected to disrupt hormonal homeostasis, and thus adverse effect of pesticides on reproductive and endocrine systems should be studied. Anderson et al. (19) tested 24 pesticides in vitro for interactions with the estrogen receptor (ER) and the androgen receptor (AR) in transactivation assays. Estrogen-like effects on MCF-7 cell proliferation and effects on CYP19 aromatase activity in human placental microsomes were detected. Estrogenic properties of test compounds could be estimated by measuring mRNA levels of ER $\alpha$ and ER $\beta$ (20). In vitro reporter gene assays using Chinese hamster ovary cells and inhibition of CYP19 aromatase activity seemed to be powerful screening tools for reproductive disruption by many pesticides (21).

Selected different classes of pesticides have been reported to have genotoxicity and mutagenicity. Thus, investigators have designed in vitro tests for these effects. The in vitro sister chromatid exchange (SCE) assay in human lymphocytes was suggested as a sensitive method of measuring the potential mutagenicity/carcinogenicity of pesticides (22). DNA damages by pesticides also could be estimated by measuring chromosomal aberrations (chromatid breaks, fragments, gaps, aneuploidy, and satellite association) and standard alkaline comet assay on human peripheral lymphocytes in vitro. The experimental results suggested that analysis of genotoxicity may serve as an important indicator of the risks of occupational and household exposure to pesticides (23-25). Due to the complex metabolic competence, the chicken embryo may serve as a standard model system for genotoxicity testing, owing to its capability of undergoing metabolic activation and deactivation of xenobiotics. The micronucleus test and the comet assay using the chicken embryo can be performed to evaluate the genotoxic potential of insecticides $(26,27)$.

Some pesticides are hematotoxic and cause agranulocytosis, neutropenia, thrombopenia and anemia. Parent-Massin and Thouvenot (28) had performed pesticide hematotoxicity, using human and rat progenitor cells, human and rat colony-forming unit-granulocyte and macrophage (CFU-GM), known to be either hematotoxic or innocuous for blood cells. Their results demonstrated that pesticides known to be hematotoxic in vivo inhibited the development of progenitors in vitro. Such techniques, therefore, could be used in predictive and retrospective studies to identify chemicals responsible for hematotoxicity.

Many classes of insecticides, such as organophosphates and carbamates, have neurotoxic potential. Therefore, alternative neurotoxicity tests are essential for risk assessment of such insecticides. Many researchers have determined the inhibitory power $\left(\mathrm{I}_{50}\right)$ of a number of compounds against acetylcholine esterase and neurotoxic esterase - inhibition of which is known to cause neurotoxicity - of hen and human brain in vitro. Comparison of the ratio of these $\mathrm{I}_{50}$ 's with previous in vivo toxicity data suggested that this ratio has predictive value for sensitivity to insecticides (29). Cell cultures derived from nervous system tissue have proven to be powerful tools for elucidating cellular and molecular mechanisms of nervous system development and function, and have been used to understand the mechanism of action of neurotoxic chemicals. Recently, it has been suggested that in vitro models could be used to screen for chemical effects on critical cellular events during neurodevelopment, including differentiation and neurite growth. Reports in the literature describe cell culture systems that are used to quantify the effects of pesticides on neurodevelopmental processes in vitro by examining their effects on neurite outgrowth (30).

As an alternative to the standard Draize eye irritancy test, the potential irritancy of compounds can be evaluated by observing adverse changes that occur in the chorioallantoic membrane (CAM) of the hen egg after exposure to pesticides. A good correlation was found between this CAM assay and the in vivo Draize eye irritation test (31). Thus, it could be a valid predictor of irritation and/or corrosion induced by pesticides in human eyes. This assay represents an in vitro alternative method that is expected to reduce public concerns, required experimenter's time, and use of rabbits for pesticide assessment.

Artificial human three-dimensional skin models have gradually replaced in vivo skin irritation and sensitization tests in recent years. In particular, toxicologists are applying such models to toxicity tests for cosmetics. A three-dimensional human skin model consisting of several layers of actively dividing and metabolically active human neonatal foreskin-derived fibroblasts and epidermal keratinocytes grown on nylon mesh has been used to assess the in vitro toxicity of pesticides (32). Nowadays, few companies construct and sell human skin models such as KeraSkin ${ }^{\mathrm{TM}}$, EpiSkin ${ }^{\mathrm{TM}}$, and MelaSkin ${ }^{\mathrm{TM}}$. Thus, many investigators are using these models for pesticide testing. Jeong et al. (33) studies the effects of several pesticides on KeraSkin ${ }^{\mathrm{TM}}$ and measured the cell viability in the culture medium collected after treatment with pesticides for $24 \sim 72 \mathrm{hrs}$. Changes in cell viability caused by the formulation were taken as predictors of skin irritation and results suggested that alternative testing methods using the Keraskin ${ }^{\mathrm{TM}}$ model could be used to evaluate pesticide toxicity equivalent to a primary irritation index (P.I.I.) score of greater than or equal to 2.1. Further studies of pesticide skin irritation using alternative methods are necessary to validate these improved methods of alternative pesticide safety evaluation(33). As for cosmetics industry, it is anticipated that artificial 3D skin models may entirely replace animal skin testing in pesticide industry for 
release and permission of new products, mechanism studies and risk assessment.

Recently, a neutral red uptake (NRU) assay with epithelioma papulosum cyprini (EPC) cell was developed as an alternative to the fish acute toxicity test. The epithelioma papulosum cyprini (EPC) cell is originated from the epidermal tissue of Cyprinus carpio (common carp). Results from in vivo fish acute toxicity tests conducted according to OECD Test Guideline No. 203 using Aphyocypris chinensis, Oryzias latipes, and C. carpio. suggested that the EPC cell viability assay represents a viable alternative to the fish acute toxicity test, owing to the good correlation between these tests and the NRU assay is expected to serve as a useful tool for the prediction of acute fish lethality for pesticides (34). However, further studies with a larger set of pesticides are needed to strengthen the reliability of the assays and to validate the correlation with in vivo data (34).

\section{IN VIVO ALTERNATIVE PESTICIDE TOXICITY TESTING METHODS}

Alternative toxicity testing methods using living organism indicate the replacement of animals such as vertebrates with animals that are lower on the phylogenetic scale, modifications of husbandry or experimental procedures to enhance animal well-being and minimize or eliminate pain and distress and the use of fewer animals. The 'Guide for the Care and Use of Laboratory Animals' plays an important role in decision making regarding the use of vertebrate laboratory animals because it establishes the minimum ethical, practice, and care standards for researchers and their institutions (35).

As animal-replacing methods, fishes, amphibians such as the tadpole, or invertebrates such as the sea urchin could be taken advantage of pesticides toxicity testing. They have lots of advantages. That is to say, these animals can be applied in various conditions, bred and manipulated easily, and are available in many different species and sizes. Also, they can be used in various field of pesticide toxicity such as embryology, neurobiology, endocrinology, and carcinogenesis. Rudek et al. (36) demonstrated that pyrethroid causes mitotic spindle damage, clastogenic activity and micronucleated red blood cells using tadpoles (Rana temporaria and Xenopus laevis). The micronucleus test in amphibians represents an excellent tool for alternative testing and monitoring of pesticide toxicity in natural water.

The fish embryo toxicity (FET) test has been proposed as an alternative to the acute fish test. It can be used to collect data on various types of environmental assessments (e.g., hazard, risk, effluent, and classification and labeling of chemicals). The establishment of definitive testing strategies and procedures for a fish eleutheroembryo toxicity (FEET) test is in progress and will provide another option for acute test- ing of juvenile or adult fish (as in OECD 203 and similar guidelines) in the near future (37).

Invertebrates usually raise less societal concern than mammals, birds, or even lower vertebrates such as fish. Invertebrate species seem to be useful animal models to link, in a mechanistic way, suborganismal effects of environmental chemicals to changes at the population and community levels. Thus, replacement of vertebrates with invertebrates in ecotoxicity testing of pesticides is quite feasible. In laboratory tests or field studies, the actual or potential value of invertebrates as alternatives is evaluated from their ability to replace or prevent the use of vertebrates. For example, the study of the mechanism of action of pyrethroid was performed using various experimental models, including squid giant axon, crayfish giant axon and stretch receptor organ, snail neurons, cockroach nerve cord and giant axon, and isolated insect neurons (38). Some insecticides and herbicides were investigated using an embryogenesis bioassay in the sea urchin and other marine invertebrates, and it was demonstrated that the procedure toxicity testing on non-target marine species and for the assessment of the biological quality of marine water (39).

However, the most important problem facing species replacement is that of extrapolation of responses to environmental chemicals from invertebrates to vertebrates (including humans), which is very difficult because of the differences in pharmacokinetics, pharmacodynamics, detoxification, recovery and other factors (38). Such translation issues severely inhibit the prompt adoption of alternative testing methods and should be a focus of research in the near future.

As an example of subject reduction, OECD recommends that laboratory animal usage could be reduced by using cytotoxicity tests to estimate starting doses for acute oral systemic toxicity tests because $\mathrm{IC}_{50}$ values from in vitro basal cytotoxicity tests could be used to predict $\mathrm{LD}_{50}$ values for use as starting doses for the Acute Toxic Class (ATC) method (TG 423) or the Up-and-Down Procedure (UDP) (TG 425) test guideline. Simulations for reference substances showed that using in vitro cytotoxicity assays to estimate an $\mathrm{LD}_{50}$ to use as a starting dose could reduce animal use by up to $28 \%$ for acute oral toxicity testing, and by as much as $50 \%$ for non-classified substances $(40)$.

To predict skin toxicity, such as skin allergic reactions, or contact dermatitis, the murine local lymph node assay (LLNA) can be used based on the protocol of the ICCVAM or the OECD. The ICCVAM recommended the LLNA as a valid alternative to traditionally accepted guinea pig test methods for assessing the hazard potential in most testing situations. The National Toxicology Program Interagency Center for the Evaluation of Alternative Toxicological Methods (NICEATM) LLNA database of more than 600 substances includes data for 104 pesticide formulations. ICCVAM concludes that these data support the usefulness of the LLNA for testing pesticide formulations (41). 


\section{IN SILICO ALTERNATIVE PESTICIDE TOXICITY TESTING METHODS}

It is necessary to think creatively to break from conventional animal testing methods. A typical example is virtual environment modeling of organisms. In silico is a term created by appearance and advance in bioinformatics. It means technics that study biological phenomena and design drugs using computer simulations (42). As a result of advances in computer technology, nowadays numerous biological reactions can be modeled and predicted with high class-algorithm. The advantages of in silico tools are low cost, standardization, minimal equipment needs, minimal training requirements, and fast execution. In general, in silico tools allow for advanced biometry and have clearly defined usage scopes (16).

A strong promoter of in silico approaches was the Registration, Evaluation, Authorization and Restriction of Chemicals (REACH) legislation passed by the EU (16). Currently, some countries, under support of government, have promoted in silico projects based on massive databases and preceding in vitro, in vivo experiments. In case of the United States, the EPA launched a related program called ToxCast in 2007 which aims to screen and prioritize chemi-

Table 1. Summary of current state on alternative pesticide toxicity testing areas in this review

\begin{tabular}{|c|c|c|}
\hline & Developed Researches & Relevant regulations/guidelines \\
\hline In vitro & $\begin{array}{l}\text { Immunotoxicity tests (18) } \\
\text { Iodo-nitro-tetrazolium reductase test } \\
\text { Leukocyte migration-inhibition assay } \\
\text { Reproductive and endocrine toxicity tests } \\
\text { Interactions with estrogen receptor and androgen receptor in } \\
\text { transactivation assays (19) } \\
\text { CYP19 aromatase activity in human placental microsomes (19) } \\
\text { Measurement of mRNA levels of ER } \alpha \text { and ER } \beta \text { (20) } \\
\text { In vitro reporter gene assays (21) } \\
\text { Inhibition of CYP19 aromatase activity (21) } \\
\text { Genotoxicity/mutagenicity tests } \\
\text { Sister chromatid exchange assay (22) } \\
\text { Standard alkaline comet assay (23-25) } \\
\text { Micronucleus test and comet assay using the chicken embryo } \\
\text { (26,27) } \\
\text { Hematotoxicity tests (28) } \\
\text { Neurotoxicity tests } \\
\text { Others } \\
\text { Chorioallantoic membrane assays (31) } \\
\text { Three-dimensional skin models (32,33) } \\
\text { Neutral red uptake (NRU) assay (34) } \\
\text { High-throughput in vitro toxicity screening (HTS) (47) } \\
\text { Microfabricated platforms (56) }\end{array}$ & $\begin{array}{l}\text { Guide for the Care and Use of Laboratory Animals } \\
\text { (35) } \\
\text { International harmonization of test guidelines by the } \\
\text { OECD for chemicals/pesticides } \\
\text { Veterinary medicine and International Conference on } \\
\text { Harmonization (ICH and VICH) (16) }\end{array}$ \\
\hline In vivo & $\begin{array}{l}\text { Animal-replacing methods } \\
\text { Fishes, amphibians, invertebrates } \\
\text { Mitotic spindle damage, Clastogenic activity and Micronucleus } \\
\text { test using tadpoles (36) } \\
\text { Fish embryo toxicity (FET) test (37) } \\
\text { Using neurons and axons of invertebrates (38) } \\
\text { Embryogenesis bioassay in the sea urchin and marine } \\
\text { invertebrates (39) } \\
\text { Murine local lymph node assay (41) }\end{array}$ & $\begin{array}{l}\text { Guide for the Care and Use of Laboratory Animals } \\
\text { (35) } \\
\text { OECD Guidelines for the Testing of Chemicals, } \\
\text { Section 4, Test No. } 420 \text { (10) } \\
\text { OECD Guidelines for the Testing of Chemicals, } \\
\text { Section 4, Test No. } 423 \text { (11) } \\
\text { OECD Guidelines for the Testing of Chemicals, } \\
\text { Section 4, Test No. } 425 \text { (40) }\end{array}$ \\
\hline In silico & $\begin{array}{l}\text { ToxCast program (USA) (43) } \\
\text { Establishment of Center for cost-effective cell-based and } \\
\text { computational toxicity evaluation systems (Korea) (44) } \\
\text { Developing a toxicity evaluation system using virtual cells (Japan) } \\
\text { (44) } \\
\text { Functional genomics }(45,46) \\
\text { Structural genomics }(45) \\
\text { Structure-activity relationship analysis }\end{array}$ & $\begin{array}{l}\text { Registration, Evaluation, Authorization and } \\
\text { Restriction of Chemicals (REACH) legislation (16) }\end{array}$ \\
\hline
\end{tabular}


cals for potential human toxicity using in vitro assays and in silico approaches. The first phase of ToxCast tested 309 active chemicals (mostly pesticide) in 467 assays across 9 technologies, including high-throughput cell-free and cellbased assays, in multiple human primary cells and cell lines. ToxCast has evaluated over 2,000 chemicals and they were evaluated in over 700 high-throughput assays that covered a range of high-level cellular responses and approximately 300 signaling pathways. The large ToxCast data set, which links in vitro and in vivo assay, results to genes and pathways, providing a unique public resource for researchers modeling chemical biology and toxicity. ToxCast expects to continue to provide innovative computational methods for evaluating potential health impacts of environmental chemicals (43).

In Korea, the National Institute of Food and Drug Safety Evaluation in the Ministry of Food and Drug Safety (MFDS) constituted a center for cost-effective cell-based and computational toxicity evaluation systems in 2010 , which is developing eco-friendly technologies to evaluate and predict toxic responses in cells or tissues using molecular biology. More than 150 million laboratory mice are used per year for toxicity testing in Korea. The adoption of in silico toxicity evaluation methods is expected by the MFDS to reduce the use of laboratory animals to less than half of its current rate. This change corresponds with a global trend to downscale animal use in toxicological studies for reasons of ethics and high cost. The center has developed molecular screening technology, in silico methods of toxicity prediction, and biomarkers of target organ toxicity. In addition, a research institute that is part of the Ministry of Health, Labour and Welfare (MHLW) of Japan, has developed a toxicity evaluation system using virtual cells (44).

Although machines cannot perfectly represent living of laboratory animals, computer models could prevent the unnecessary use of millions of animals in painful toxicity tests. Furthermore, in silico methods could be valuable as alternative test for screening the potential risk of many pesticides to public health.

\section{POSSIBLE METHODS WIDELY APPLICABLE TO ALTERNATIVE TESTING OF PESTICIDES}

With an advances in modern science like as biotechnology and "-omics" (genomics, proteomics, etc.), numerous alternative approaches have been investigated and established. There are some beneficial and applicable tools that are expected to be utilized widely in agrochemical evaluation.

Functional genomics represents a new phase of genome analysis. It provides a fertile ground for creative thinking to develop innovative technologies that make use of the vast repository of structural genomics information. Specifically, functional genomics refers to the development and application of global (genome-wide or system-wide) experimental approaches to assess gene function by making use of the information and reagents provided by structural genomics (45). It is characterized by high throughput or large scale experimental methodologies that are combined with statistical and computational analysis of the results. Computational biology performs a critical and expanding role in this area, and functional genomics is characterized by mining of data sets for particularly valuable information. It promises to rapidly narrow the gap between the understanding of sequence and function and to yield new insights into the behavior of biological systems (45). One of good example on pesticide toxicity is functional genomics of the human paraoxonase polymorphisms that levels and genetic variability of the PON1 position 192 isoforms (Gln/Arg) influence sensitivity to specific insecticides or nerve agents (46). More investigation in this area is definitely needed to be pursued.

High-throughput in vitro toxicity screening (HTS) was initially developed by the pharmaceutical industry (47). HTS can provide an efficient way to identify potential biological targets for chemicals. The combination of rapid in vitro assays would allow for more efficient risk assessment for a single compound and the accrual of generic information on chemical characteristics for pathway activation to allow for the prediction of the effects (48). The transition to a new toxicity testing paradigm that relies heavily on in vitro HTS assays will require a parallel investment to characterize the pharmacokinetics and exposure levels of these chemicals. These parallel efforts will add valuable information on parameters critical to interpreting the biologically relevant exposure scenarios that should yield more informative and effective prioritization models (49).

The concept of evidence-based medicine (EBM) has emerged from clinical medicine, which retrospectively assesses the evidence of adequacy of a given approach. EBM advocates have developed procedures, including meta-analysis, to collect and evaluate all the available evidence in case where definitive studies are unavailable (50). To answer questions about general and specific causation, Guzelian et al. (51) have constructed a framework for evidence-based toxicology (EBT) that is derived from the accepted principles of EBM, and have suggested new toxicological approaches. We customarily distinguish between known relationships (facts) and those propositions that are only possible or partially known (suppositions). EBT represents an objective, systematic method for classifying these relationships as potential or established, based on accumulated knowledge. EBT provides a uniform, structured guide for evaluating quantitative and judgmental aspects of the elements of a causational analysis (51). EBT promises to allow for informed decision making in risk assessment for individual compounds for which several studies are available. For this purpose, the quality score concept for toxicological studies needs to be further developed.

Physiologically-based pharmacokinetic (PBPK) model- 
ing refers to the development of quantitative descriptions of the absorption, distribution, metabolism and elimination of chemicals, on the basis of interrelationships among the critical determinants of these processes. PBPK models predict the time-course of parent chemical, metabolite(s) or biomarkers in the exposed organism (52). Some organophosphates have been investigated using PBPK methods. The PBPK model, developed for chlorpyrifos and diazinon, was capable of quantifying target tissue dosimetry and dynamic responses in rats and humans and, thus, could be used to link metabolism to cholinesterase inhibition across species. Furthermore, it could be used to quantitatively assess the risk associated with exposure, as well as to design and focus of future experimental research $(53,54)$. An advantage of PBPK models is that they can be adapted to newer insights, and the knowledge gained from the modeled processes and structures can be expected to increase the model's predictive and explanatory value (55). PBPK models provide a theoretical basis for integrating in vitro data and making tissue dosimetry predictions in the animal, thus, allowing for the reduction or refinement of animal use in pharmacokinetic and toxicity studies. PBPK models of other pesticides are urgently needed to facilitate the reduction of animal use.

Sharma et al. (56) suggested the use of microfabricated platforms that the disciplines of microscale engineering in conjugation with tissue engineering could potentially provide such a platform and revolutionize the in vitro allergy test field. There are several advantages in utilizing microdevices for cell culture. Scaling down to the micron level permits material saving and evaluating responses at a single cell level. Microdevices can simulate interconnected cells found in vivo and microfluidics can reproduce in vivo like channel sizes. Thus, this type of model provides further methods for alternative toxicity testing and presumed predictability (56).

In addition to the methods described herein, there are other alternative toxicity testing tools that deserve consideration, such as structure-activity relationship analysis, biomarkers, and so on. Because of the limitation of each alternative approaches, to obtain the most precise and valid results, we recommend that several complementary methods had better be used. Greater reliance on these alternative techniques for pesticide testing will reduce the need for animals, assuage ethical concerns, and, then, positively energize pesticide toxicology.

\section{CONCLUSIONS AND EXPECTED FUTURE OF PESTICIDE TOXICOLOGY}

Twenty-one century is the era of agriculture and environment. Among these, the beneficial potential of pesticides makes their use inevitable. Therefore, effective testing of the toxicity of agrochemicals is needed to support the development of low-toxicity pesticides. However, animal testing is costly and engenders ethical concerns. Therefore, it is important to develop non-animal toxicological methods for pesticides that allow for the collection of critical safety data through alternative approaches.

Many issues with alternative toxicity test for agrochemicals remain to be resolved, primarily with regard to some potential limitations and validation of the alternative methodology. Furthermore, extrapolation from dose and exposure of pesticides on cells or animals to real dose and exposure of pesticides on humans or ecosystem could be complicated (4). Thus, it is necessary to secure the validation and overcome the limitations of pesticides alternative toxicity tests. Regulatory agencies and the users of alternative test systems need a process to ensure that "me-too" test kits developed according to the generic descriptions produce results similar to those obtained using the system originally validated and accepted (4). Continued refinement of the in vitro assays to better reflect in vivo adverse effects and improvement of in vitro pharmacokinetic assays will eventually allow researchers to move beyond hazard-based prioritization to risk assessment (49).

Experts-in-these fields estimate that the replacement of animal experiments for most aspects of skin sensitization and toxicokinetics will be possible within 5 9 years, and that within 10 years, this replacement will be required in other areas (17). Although laboratory animals are indispensable in many scientific fields, it is very important that persistent efforts should be focused to minimize animal use, and to apply alternative testing methods in pesticide safety evaluation that also reduce study time and cost.

Pesticide toxicology will benefit greatly from the use of the robust validated alternative methods described herein and the development of novel techniques. If reliable alternative methods for pesticide toxicity evaluation are established and they reflect toxic pathway by actual exposure of each agrochemicals, we could not only satisfy $3 \mathrm{R}$ principles and humanity for animals weaker than human but also protect/improve public health and ecosystem. Ultimately, an integrated viewpoint encompassing circumstances of humans, veterinary animals and the environment will advance alternative pesticide toxicology area.

\section{ACKNOWLEDGEMENTS}

Part of this work is supported by "Cooperative Research Program for Agriculture Science \& Technology Development (Project title: Development of alternative test for systemic toxicity and network analysis of toxicity parameters, Project No. PJ00995002)" Rural Development Administration, Republic of Korea.

\section{REFERENCES}

1. Korea Ministry of Government Legislation. (2014) Agricul- 
tural chemicals regulation law. http://www.law.go.kr/lsInfoP.do?1siSeq=152000\&efYd=20140318\#0000.

2. Korea Crop Protection Association. (2005) Safety of Pesticides - A Great Difference between Original Toxicity and Product Toxicity. Life and Pesticides, March, pp. 38-39.

3. Russell, W.M.S., Burch, R.L. and Hume, C.W. (1959) The principles of humane experimental technique, Methuen, London.

4. Gupta, K., Rispin, A., Stitzel, K., Coecke, S. and Harbell, J. (2005) Ensuring quality of in vitro alternative test methods: Issues and answers. Regul. Toxicol. Pharmacol., 43, 219-224.

5. Korea Ministry of Government Legislation. (2014) Animal Protection Law.

http://www.law.go.kr/lsInfoP.do?lsiSeq=152326\&efYd= 20140324\#0000.

6. Notification No. 2013-21 of Rural Development Administration, Partly Amendment of Registration Standard of Pesticides and Technical Ingredients.

7. Rural Development Administration. (2012) Press materialReducing Animals on Evaluation Tests of Pesticides Safety.

8. Beyond Pesticides, Bee protective : Pollinators and Pesticides, What the Science.

http://www.beyondpesticides.org/pollinators/research.php.

9. Johnson, R.M., Ellis, M.D., Mullin, C.A. and Frazier, M. (2010) Pesticides and honeybee toxicity-USA. Apidologie, 41, 312-331.

10. OECD. (2001) Test No. 420: Acute Oral Toxicity - Fixed Dose Procedure. OECD, pp. 1-14.

11. OECD. (2001) Test No. 423: Acute Oral toxicity - Acute Toxic Class Method. OECD, pp. 1-14.

12. Park, K.H. (2011) Pesticides toxicity test system management and management condition. Annu. Meet. Korean Soc. Pestic. Sci., 30-37.

13. European Commission. (2013) Legislation on pesticides-regulation. European, 283, 284.

14. EPA. Federal Insecticide, Fungicide, and Rodenticide Act (FIFRA). U.S. Environmental Protection Agency, http:// www.epa.gov/oecaagct/lfra.html.

15. EPA. (2012) Pesticide Program Dialogue Committee 21st Century Toxicology/New Integrated Testing Strategies Workgroup. U.S. Environmental Protection Agency.

16. Hartung, T. (2011) From alternative methods to a new toxicology. Eur. J. Pharm. Biopharm., 77, 338-349.

17. Araújo, G.L.D., Campos, M.A.A., Valente, M.A.S., Silva, S.C.T., Franca, F.D., Chaves, M.M. and Tagliati, C.A. (2014) Alternative methods in toxicity testing: the current approach. Braz. J. Pharm. Sci., 50, 55-62.

18. Pistl, J., Kovalkovičová, N., Holovská, V., Legáth, J. and Mikula, I. (2003) Determination of the immunotoxic potential of pesticides on functional activity of sheep leukocytes in vitro. Toxicology, 188, 73-81.

19. Andersen, H.R., Vinggaard, A.M., Rasmussen, T.H., Gjermandsen, I.M. and Bonefeld-Jørgensen, E.C. (2002) Effects of Currently Used Pesticides in Assays for Estrogenicity, Androgenicity, and Aromatase Activity In Vitro. Toxicol. Appl. Pharmacol., 179, 1-12.

20. Grünfeld, H.T. and Bonefeld-Jorgensen, E.C. (2004) Effect of in vitro estrogenic pesticides on human oestrogen receptor $\alpha$ and $\beta$ mRNA levels. Toxicol. Lett., 151, 467-480.
21. Kojima, H., Katsura, E., Takeuchi, S., Niiyama, K. and Kobayashi, K. (2004) Screening for estrogen and androgen receptor activities in 200 pesticides by in vitro reporter gene assays using Chinese hamster ovary cells. Environ. Health Perspect., 112, 524-531.

22. Vigfusson, N.V. and Vyse, E.R. (1980) The effect of the pesticides, Dexon, Captan and Roundup, on sister-chromatid exchanges in human lymphocytes in vitro. Mutat. Res., 79, 5357.

23. Jamil, K., Shaik, A.P., Mahboob, M. and Krishna, D. (2004) Effect of organophosphorus and organochlorine pesticides (monochrotophos, chlorpyriphos, dimethoate, and endosulfan) on human lymphocytes in-vitro. Drug Chem. Toxicol., 27, 133-144.

24. Ündeğer, Ü. and Baçaran, N. (2005) Effects of pesticides on human peripheral lymphocytes in vitro: induction of DNA damage. Arch. Toxicol., 79, 169-176.

25. Das, P.P., Shaik, A.P. and Jamil, K. (2007) Genotoxicity induced by pesticide mixtures: in-vitro studies on human peripheral blood lymphocytes. Toxicol. Ind. Health, 23, 449458.

26. Vinggaard, A.M., Hnida, C., Breinholt, V. and Larsen, J.C. (2000) Screening of selected pesticides for inhibition of CYP19 aromatase activity in vitro. Toxicol. In vitro, 14, 227234.

27. Uggini, G.K. and Suresh, B. (2013) Genotoxic effects of two different classes of insecticide in developing chick embryos. Toxicol. Environ. Chem., 95, 992-1005.

28. Parent-Massin, D. and Thouvenot, D. (1993) In vitro study of pesticide hematotoxicity in human and rat progenitors. $J$. Pharmacol. Toxicol. Methods, 30, 203-207.

29. Lotti, M. and Johnson, M.K. (1978) Neurotoxicity of organophosphorus pesticides: predictions can be based on in vitro studies with hen and human enzymes. Arch. Toxicol., 41, 215221.

30. Radio, N.M. and Mundy, W.R. (2008) Developmental neurotoxicity testing in vitro : Models for assessing chemical effects on neurite outgrowth. Neurotoxicology, 29, 361-376.

31. Kishore, A.S., Surekha, P.A., Sekhar, P.V., Srinivas, A. and Murthy, P.B. (2008) Hen egg chorioallantoic membrane bioassay: an in vitro alternative to draize eye irritation test for pesticide screening. Int. J. Toxicol., 27, 449-453.

32. Triglia, D., Braa, S.S., Yonan, C. and Naughton, G.K. (1991) In vitro toxicity of various classes of test agents using the neutral red assay on a human three-dimensional physiologic skin model. In Vitro Cell. Dev. Biol. Anim., 27, 239-244.

33. Jeong, M.H., Kim, M.K., Park, S.J., You, A.S., Hong, S.S., Park, K.H. and Park, J.E. (2012) The application of alternative methods for skin irritation evaluation on pesticides. Korean J. Pestic. Sci., 16, 261-266.

34. Seo, J.H., Park, J.W., Lee, S.K. and Kim, W.K. (2014) Application of neutral red uptake assay using EPC cells as an alternative to the fish acute toxicity test for pesticide. Korean $\mathrm{J}$. Pestic. Sci., 18, 8-13.

35. National Research Council of the National Academies. (2011) Guide for the Care and Use of Laboratory Animals (8th edition), the National academies Press, Washington D.C, pp. 1-8.

36. Rudek, Z. and Rożek, M. (1992) Induction of micronuclei in tadpoles of Rana temporaria and Xenopus laevis by the pyre- 
throid Fastac 10 EC. Mutat.Res., 298, 25-29.

37. Embry, M.R., Belangerb, S.E., Braunbeckc, T.A., Galay-Burgosd, M., Haldere, M., Hintonf, D.E., Léonardg, M.A., Lillicrap, A., Norberg-Kingi, T. and Whalej, G. (2010) The fish embryo toxicity test as an animal alternative method in hazard and risk assessment and scientific research. Aquat. Toxicol., 97, 79-87.

38. Lagadic, L. and Caquet, T. (1998) Invertebrates in testing of environmental chemicals: Are they alternatives? Environ. Health Perspect., 106 Suppl 2, 593-611.

39. Bellas, J., Beiras, R., Mariño-Balsa, J.C. and Fernández, N. (2005) Toxicity of organic compounds to marine invertebrate embryos and larvae: a comparison between the sea urchin embryogenesis bioassay and alternative test species. Ecotoxicology, 14, 337-353.

40. OECD. (2010) Guidance document on using cytotoxicity tests to estimate starting doses for acute oral systemic toxicity tests. OECD, France, pp. 1-54.

41. McMahon, T., McCall, D., Matheson. J., Jacobs, A., Burns, T., Allen, D. and Stokes, W. (2011) Evaluation of the Murine Local Lymph Node Assay (LLNA) for Assessing the Allergic Contact Dermatitis Hazard Potential of Pesticide Formulations. Niceatm-Iccvam 8th World Congress Poster, USA, pp. 1.

42. Doosan encyclopedia. http://www.doopedia.co.kr, 'In silico'.

43. Judson, R.S., Houck, K.A., Kavlock, R.J., Knudsen, T.B., Martin, M.T., Mortensen, H.M., Reif, D.M., Rotroff, D.M., Shah, I., Richard, A.M. and Dix, D.J. (2010) In vitro screening of environmental chemicals for targeted testing prioritization: the ToxCast project. Environ. Health Perspect., 118, 485492.

44. Korea Food And Drug Administration. (2011) Press material KFDA hosted international symposium on computational toxicity evaluation system. Korea.

45. Hieter, P. and Boguski, M. (1997) Functional genomics: it's all how you read it. Science, 278, 601-602.

46. Costa, L.G., Cole, T.B., Jarvik, G.P. and Furlong, C.E. (2003) Functional genomics of the paraoxonase (PON1) polymorphisms: effects on pesticide sensitivity, cardiovascular disease, and drug metabolism. Annu. Rev. Med., 54, 371-392.

47. Houck, K.A. and Kavlock, R.J. (2008) Understanding mecha- nisms of toxicity: Insights from drug discovery research. Toxicol. Appl. Pharmacol., 227, 163-178.

48. Krewski, D., Westphal, M., Al-Zoughool, M., Croteau, M.C. and Andersen, M.E. (2011) New directions in toxicity testing. Annu. Rev. Public Health, 32, 161-178.

49. Wetmore, B.A., Wambaugh, J.F., Ferguson, S.S., Sochaski, M.A., Rotroff, D.M., Freeman, K., Clewell, H.J. 3rd., Dix, D.J., Andersen, M.E., Houck, K.A., Allen, B., Judson, R.S., Singh, R., Kavlock, R.J., Richard, A.M. and Thomas, R.S. (2012) Integration of dosimetry, exposure, and high-throughput screening data in chemical toxicity assessment. Toxicol. Sci., 125, 157-174.

50. Hartung, T. (2010) Evidence-based toxicology-The toolbox of validation for the 21st century? ALTEX, 27, 253-263.

51. Guzelian, P.S., Victoroff, M.S., Halmes, N.C., James, R.C. and Guzelian, C.P. (2005) Evidence-based toxicology: a comprehensive framework for causation. Hum. Exp. Toxicol., 24, 161-201.

52. Balls, M., Combes, R.D. and Bhogal, N. (2012) Physiologically-based pharmacokinetic (PBPK) models in toxicity testing and risk in New Technologies for Toxicity Testing (Lipscomb, J.C., Haddad, S., Poet, T., Krishnan, K.). Springer, New York, pp. 76-95.

53. Timchalk, C., Nolan, R.J., Mendrala, A.L., Dittenber, D.A., Brzak, K.A. and Mattsson, J.L. (2002) A physiologically based pharmacokinetic and pharmacodynamic (PBPK/PD) model for the organophosphate insecticide chlorpyrifos in rats and humans. Toxicol. Sci., 66, 34-53.

54. Poet, T.S., Kousba, A.A., Dennison, S.L. and Timchalk, C. (2004) Physiologically based pharmacokinetic/pharmacodynamic model for the organophosphorus pesticide diazinon. Neurotoxicology, 25, 1013-1030.

55. van der Merwe, D., Brooks, J.D., Gehring, R., Baynes, R.E., Monteiro-Riviere, N.A. and Riviere, J.E. (2006) A physiologically based pharmacokinetic model of organophosphate dermal absorption. Toxicol. Sci., 89, 188-204.

56. Sharma, N.S., Jindal, R., Mitra, B., Lee, S., Li, L., Maguire, T.J., Schloss, R. and Yarmush, M.L. (2012) Perspectives on Non-Animal Alternatives for Assessing Sensitization Potential in Allergic Contact Dermatitis. Cell. Mol. Bioeng., 5, 5272. 